\title{
Almost Conservative Four-Dimensional Matrices through de la Vallée-Poussin Mean
}

\author{
S. A. Mohiuddine and Abdullah Alotaibi \\ Department of Mathematics, Faculty of Science, King Abdulaziz University, P.O. Box 80203, Jeddah 21589, Saudi Arabia
}

Correspondence should be addressed to S. A. Mohiuddine; mohiuddine@gmail.com

Received 1 November 2013; Accepted 17 December 2013; Published 2 January 2014

Academic Editor: Mohammad Mursaleen

Copyright (C) 2014 S. A. Mohiuddine and A. Alotaibi. This is an open access article distributed under the Creative Commons Attribution License, which permits unrestricted use, distribution, and reproduction in any medium, provided the original work is properly cited.

\begin{abstract}
The purpose of this paper is to generalize the concept of almost convergence for double sequence through the notion of de la Vallée-Poussin mean for double sequences. We also define and characterize the generalized regularly almost conservative and almost coercive four-dimensional matrices. Further, we characterize the infinite matrices which transform the sequence belonging to the space of absolutely convergent double series into the space of generalized almost convergence.
\end{abstract}

\section{Introduction and Preliminaries}

Let $l_{\infty}$ be the Banach space of real bounded sequences $x=x_{n}$ with the usual norm $\|x\|=\sup \left|x_{n}\right|$. There exist continuous linear functionals on $l_{\infty}$ called Banach limits [1]. It is well known that any Banach limit of $x$ lies between lim inf $x$ and $\lim \sup x$. The idea of almost convergence of Lorentz [2] is narrowly connected with the limits of S. Banach; that is, a sequence $x_{n} \in l_{\infty}$ is almost convergent to $l$ if all of its Banach limits are equal. As an application of almost convergence, Mohiuddine [3] obtained some approximation theorems for sequence of positive linear operator through this notion. For double sequence, the notion of almost convergence was first introduced by Móricz and Rhoades [4]. The authors of [5] introduced the notion of Banach limit for double sequence and characterized the spaces of almost and strong almost convergence for double sequences through some sublinear functionals. For more details on these concepts, one can refer to $[6-12]$.

We say that a double sequence $x=\left(x_{j, k}: j, k=0,1\right.$, $2, \ldots)$ of real or complex numbers is bounded if

$$
\|x\|=\sup _{j, k}\left|x_{j, k}\right|<\infty,
$$

denoted by $\mathscr{L}_{\infty}$, the space of all bounded sequence $\left(x_{j, k}\right)$.

A double sequence $x=\left(x_{j, k}\right)$ of reals is called convergent to some number $L$ in Pringsheim's sense (briefly, P-convergent to $L$ ) [13] if for every $\epsilon>0$ there exists $N \in \mathbb{N}$ such that $\left|x_{j, k}-L\right|<\epsilon$ whenever $j, k \geq N$, where $\mathbb{N}:=\{1,2,3, \ldots\}$.

If a double sequence $x=\left(x_{j, k}\right)$ in $\mathscr{L}_{\infty}$ and $x$ is also $P$ convergent to $L$, then we say that it is boundedly $P$-convergent to $L$ (briefly, $B P$-convergent to $L$ ).

A double sequence $x=\left(x_{j k}\right)$ is said to converge regularly to $L$ (briefly, $R$-convergent to $L$ ) if $x$ is converges in Pringsheim's sense, and the limits $x^{j}:=\lim _{k} x_{j k}(j \in \mathbb{N})$ and $x^{k}:=\lim _{j} x_{j k}(k \in \mathbb{N})$ exist. Note that in this case the limits $\lim _{j} \lim _{k} x_{j k}$ and $\lim _{k} \lim _{j} x_{j k}$ exist and are equal to the $P$-limit of $x$.

Throughout this paper, by $\mathscr{C}_{P}, \mathscr{C}_{B P}$, and $\mathscr{C}_{R}$, we denote the space of all $P$-convergent, $B P$-convergent, and $R$ convergent double sequences, respectively. Also, the linear space of all continuous linear functionals on $\mathscr{C}_{R}$ is denoted by $\mathscr{C}_{R}^{\prime}$.

Let $B=\left(b_{p, q, j, k}: j, k=0,1,2, \ldots\right)$ be a four-dimensional infinite matrix of real numbers for all $p, q=0,1,2, \ldots$ and $S_{1}$ a space of double sequences. Let $S_{2}$ be a double sequences space, converging with respect to a convergence rule $v \in$ $\{P, B P, R\}$. Define

$$
\begin{aligned}
S_{1}^{B, \nu}= & \left\{x=\left(x_{j, k}\right): B x=\left(B_{p, q}(x)\right)\right. \\
& \left.=v-\sum_{j, k} b_{p, q, j, k} x_{j, k} \text { exists and } B x \in S_{1}\right\} .
\end{aligned}
$$


Then, we say that a four-dimensional matrix $B$ maps the space $S_{2}$ into the space $S_{1}$ if $S_{2} \subset S_{1}^{B, v}$ and is denoted by $\left(S_{2}, S_{1}\right)$.

Móricz and Rhoades [4] extended the notion of almost convergence from single to double sequence and characterized some matrix classes involving this concept. A double sequence $x=\left(x_{j, k}\right)$ of real numbers is said to be almost convergent to a number $L$ if

$$
\lim _{p, q \rightarrow \infty} \sup _{m, n>0}\left|\frac{1}{p q} \sum_{j=m}^{m+p-1} \sum_{k=n}^{n+q-1} x_{j, k}-L\right|=0 .
$$

For more details on double sequences and 4-dimensional matrices, one can refer to [14-20].

Using the notion of almost convergence for single sequence, King [21] introduced a slightly more general class of matrices than the conservative and regular matrices, that is, almost conservative and almost regular matrices, and presented its characterization. In [22], Schaefer presented some interesting characterization for almost convergence. The Steinhaus-type theorem for the concepts of almost regular and almost coercive matrices was proved by Başar and Solak [23]. In this paper, we generalize the concept of almost convergence for double sequences with the help of double generalized de la Vallée-Poussin mean and called it $(\Lambda)$ almost convergence. Using this concept, we define the notions of regularly $(\Lambda)$ almost conservative and $(\Lambda)$ almost coercive four-dimensional matrices and obtain their necessity and sufficient conditions. Further, we introduce the space $\mathscr{L}_{1}$ of all absolutely convergent double series and characterize the matrix class $\left(\mathscr{L}_{1}, \mathscr{F}_{\Lambda}\right)$, where $\mathscr{F}_{\Lambda}$ denotes the space of $(\Lambda)$ almost convergence for double sequences.

\section{Main Results}

Definition 1. Let $\lambda=\left(\lambda_{m}: m=0,1,2, \ldots\right)$ and $\mu=\left(\mu_{n}: n=\right.$ $0,1,2, \ldots)$ be two nondecreasing sequences of positive reals with each tending to $\infty$ such that $\lambda_{m+1} \leq \lambda_{m}+1, \lambda_{1}=0$, $\mu_{n+1} \leq \mu_{n}+1, \mu_{1}=0$, and

$$
\mathfrak{\Im}_{m, n}(x)=\frac{1}{\lambda_{m} \mu_{n}} \sum_{j \in J_{m}} \sum_{k \in I_{n}} x_{j, k}
$$

is called the double generalized de la Vallée-Poussin mean, where $J_{m}=\left[m-\lambda_{m}+1, m\right]$ and $I_{n}=\left[n-\mu_{n}+1, n\right]$. We denote the set of all $\lambda$ and $\mu$ type sequences by using the symbol $\Lambda$.

Definition 2. A double sequence $x=\left(x_{j, k}\right)$ of reals is said to be $(\Lambda)$ almost convergent (briefly, $\mathscr{F}_{\Lambda}$-convergent) to some number $L$ if $x \in \mathscr{F}_{\lambda, \mu}$, where

$$
\begin{aligned}
\mathscr{F}_{\Lambda}=\{x & =\left(x_{j k}\right): P-\lim _{m, n \rightarrow \infty} \Omega_{m, n, s, t}(x) \\
= & \left.L \text { exists, uniformly in } s, t ; L=\mathscr{F}_{\Lambda^{-}} \lim x\right\}, \\
\Omega_{m, n, s, t} & (x)=\frac{1}{\lambda_{m} \mu_{n}} \sum_{j \in J_{m}} \sum_{k \in I_{n}} x_{j+s, k+t},
\end{aligned}
$$

denoted by $\mathscr{F}_{\Lambda}$, the space of all $(\Lambda)$ almost convergent sequences $\left(x_{j, k}\right)$. Note that $\mathscr{C}_{B P} \subset \mathscr{F}_{\Lambda} \subset \mathscr{L}_{\infty}$.
Remark 3. If we take $\lambda_{m}=m$ and $\mu_{n}=n$, then the notion of $(\Lambda)$ almost convergence reduced to almost convergence due to Móricz and Rhoades [4].

Definition 4. A four-dimensional matrix $B=\left(b_{p, q, j, k}\right)$ is said to be regularly $(\Lambda)$ almost conservative if it maps every $R$-convergent double sequence into $\mathscr{F}_{\Lambda}$-convergent double sequence; that is, $B \in\left(\mathscr{C}_{R}, \mathscr{F}_{\Lambda}\right)$. In addition, if $\mathscr{F}_{\Lambda}-\lim A x=$ $R$ - $\lim x$, then $B$ is regularly $(\Lambda)$ almost regular.

Definition 5. A matrix $B=\left(b_{p, q, j, k}\right)$ is said to be $(\Lambda)$ almost coercive if it maps every $B P$-convergent double sequence $\left(x_{j, k}\right)$ into $\mathscr{F}_{\Lambda}$-convergent double sequence, briefly, a matrix $B$ in $\left(\mathscr{C}_{B P}, \mathscr{F}_{\Lambda}\right)$.

Theorem 6. A matrix $B=\left(b_{p, q, j, k}\right)$ is regularly $(\Lambda)$ almost conservative if and only if

$\left(\mathrm{CR}_{1}\right)\|B\|=\sup _{p, q} \sum_{j, k}\left|b_{p, q, j, k}\right|<\infty$,

$\left(\mathrm{CR}_{2}\right) \lim _{m, n \rightarrow \infty} \alpha(m, n, s, t, j, k)=u_{j k}$, for each $j, k$ (uniformly in $s, t)$,

$\left(\mathrm{CR}_{3}\right) \lim _{m, n \rightarrow \infty} \sum_{k}|\alpha(m, n, s, t, j, k)|=u_{j 0}$, for each $j$ (uniformly in $s, t$ ),

$\left(\mathrm{CR}_{4}\right) \lim _{m, n \rightarrow \infty} \sum_{j}|\alpha(m, n, s, t, j, k)|=u_{0 k}$, for each $k$ (uniformly in $s, t$ ),

$\left(\mathrm{CR}_{5}\right) \lim _{m, n \rightarrow \infty} \sum_{j, k} \alpha(m, n, s, t, j, k)=u$, (uniformly in $s$, $t$ ),

where

$$
\beta(m, n, s, t, j, k)=\frac{1}{\lambda_{m} \mu_{n}} \sum_{p \in J_{m}} \sum_{q \in I_{n}} b_{p+s, q+t, j, k} .
$$

In this case, the $\mathscr{F}_{\Lambda}$-limit of $B x$ is

$$
\begin{array}{r}
\ell u+\sum_{j=0}^{\infty}\left(\ell_{j}-\ell\right) u_{j 0}+\sum_{k=0}^{\infty}\left(h_{k}-\ell\right) u_{0 k} \\
+\sum_{j=0}^{\infty} \sum_{k=0}^{\infty}\left(x_{j, k}-\ell_{j}-h_{k}-\ell\right) u_{j k},
\end{array}
$$

where $\ell=R-\lim x$.

Proof. Necessity. Suppose that $B$ is regularly $(\Lambda)$ almost conservative matrix. Fix $s, t \in \mathbb{Z}$, the set of integers. Let

$$
\Omega_{m, n, s, t}(x)=\frac{1}{\lambda_{m} \mu_{n}} \sum_{p \in J_{m}} \sum_{q \in I_{m}} \rho_{p+s, q+t}(x) \text {, }
$$

where

$$
\rho_{p, q}(x)=\sum_{j=0}^{\infty} \sum_{k=0}^{\infty} b_{p, q, j, k} x_{j, k} .
$$

It is clear that

$$
\rho_{p, q} \in \mathscr{C}_{R}^{\prime}, p, \quad q=0,1,2, \ldots
$$


Hence $\Omega_{m, n, s, t} \in \mathscr{C}_{R}^{\prime}$ for $m, n \in \mathbb{N}$. Since $B$ is regularly $(\Lambda)$ almost conservative, we have

$$
P-\lim _{m, n \rightarrow \infty} \Omega_{m, n, s, t}(x)=\Omega(x) \quad \text { (say) },
$$

uniformly in $s, t$. It follows that $\left(\Omega_{m, n, s, t}(x)\right)$ is bounded for $x \in \mathscr{C}_{R}$ and fixed $s, t$. Hence, $\left\|\Omega_{m, n, s, t}(x)\right\|$ is bounded by the uniform boundedness principle. by

For each $i, v \in \mathbb{Z}^{+}$, define the sequence $y=y(m, n, s, t)$

$$
y_{j, k}= \begin{cases}\operatorname{sgn}\left(\sum_{p \in J_{m}} \sum_{q \in I_{n}} b_{p+s, q+t, j, k}\right), & \text { if } 0 \leq j \leq i, 0 \leq k \leq v, \\ 0, & \text { if } v<k, i<j .\end{cases}
$$

Then, a double sequence $y \in \mathscr{C}_{R},\|y\|=1$, and

$$
\left|\Omega_{m, n, s, t}(y)\right|=\frac{1}{\lambda_{m} \mu_{n}} \sum_{j=0}^{i} \sum_{k=0}^{v}\left|\sum_{p \in J_{m}} \sum_{q \in I_{n}} b_{p+s, q+t, j, k}\right| .
$$

Hence

$$
\left|\Omega_{m, n, s, t}(y)\right| \leq\left\|\Omega_{m, n, s, t}\right\|\|y\|=\left\|\Omega_{m, n, s, t}\right\| .
$$

Therefore

$$
\frac{1}{\lambda_{m} \mu_{n}} \sum_{j=0}^{\infty} \sum_{k=0}^{\infty}\left|\sum_{p \in J_{m}} \sum_{q \in I_{n}} b_{p+s, q+t, j, k}\right| \leq\left\|\Omega_{m, n, s, t}\right\|,
$$

so that condition $\left(\mathrm{CR}_{1}\right)$ follows.

The sequences $F^{(b, c)}=\left(f_{j, k}^{(b, c)}\right), F^{(b)}=\left(f_{j, k}^{(b)}\right), G^{(c)}=\left(g_{j, k}^{(c)}\right)$, and $G=\left(g_{j, k}\right)$ are defined by

$$
\begin{gathered}
f_{j, k}^{(q, r)}= \begin{cases}1, & \text { if }(j, k)=(b, c), \\
0, & \text { otherwise, }\end{cases} \\
f_{j, k}^{(b)}= \begin{cases}1, & \text { if } j=b, \\
0, & \text { otherwise, }\end{cases} \\
g_{j, k}^{(c)}= \begin{cases}1, & \text { if } k=c, \\
0, & \text { otherwise, }\end{cases} \\
g_{j, k}=1, \quad \forall j, k .
\end{gathered}
$$

Since $F^{(j, k)}, F^{(j)}, G^{(k)}, G \in \mathscr{C}_{R}$, the $P$-limit of $\Omega_{m, n, s, t}\left(F^{(j, k)}\right)$, $\Omega_{m, n, s, t}\left(F^{(j)}\right), \Omega_{m, n, s, t}\left(G^{(k)}\right)$, and $\Omega_{m, n, s, t}(G)$ must exist, uniformly in $s, t$. Hence, the conditions $\left(\mathrm{CR}_{2}\right)-\left(\mathrm{CR}_{5}\right)$ must hold, respectively.

Sufficiency. Suppose that the conditions $\left(\mathrm{CR}_{1}\right)-\left(\mathrm{CR}_{5}\right)$ hold and a double sequence $x=\left(x_{j k}\right) \in \mathscr{C}_{R}$. Fix $s, t \in \mathbb{Z}$. Then

$$
\begin{gathered}
\Omega_{m, n, s, t}(x)=\frac{1}{\lambda_{m} \mu_{n}} \sum_{j=0}^{\infty} \sum_{k=0}^{\infty} \sum_{p \in J_{m}} \sum_{q \in I_{n}} b_{p+s, q+t, j, k} x_{j, k}, \\
\left|\Omega_{m, n, s, t}(x)\right| \leq \frac{1}{\lambda_{m} \mu_{n}} \sum_{j=0}^{\infty} \sum_{k=0}^{\infty}\left|\sum_{p \in J_{m}} \sum_{q \in I_{n}} b_{p+s, q+t, j, k}\right|\left\|x_{j, k}\right\| .
\end{gathered}
$$

Therefore, by $\left(\mathrm{CR}_{1}\right)$, we have $\left|\Omega_{m, n, s, t}(x)\right| \leq C_{s, t}\|x\|$, where $C_{s, t}$ is a constant independent of $m, n$. Hence $\Omega_{m, n, s, t} \in \mathscr{C}_{R}^{\prime}$ and the sequence $\left(\left\|\Omega_{m, n, s, t}\right\|\right)$ is bounded for each $s, t \in \mathbb{Z}^{+}$. It follow from the conditions $\left(\mathrm{CR}_{2}\right),\left(\mathrm{CR}_{3}\right),\left(\mathrm{CR}_{4}\right)$, and $\left(\mathrm{CR}_{5}\right)$ that the $P$-limit of $\Omega_{m, n, s, t}\left(F^{(j, k)}\right), \Omega_{m, n, s, t}\left(F^{(j)}\right), \Omega_{m, n, s, t}\left(G^{(k)}\right)$, and $\Omega_{m, n, s, t}(G)$ exist for all $j, k, s$, and $t$. Since $G, F^{(j)}, G^{(k)}$ and $F^{(j, k)}$ is a fundamental set in $\mathscr{C}_{R}$ (see [24]), it follows that

$$
\lim _{m, n \rightarrow \infty} \Omega_{m, n, s, t}(x)=\Omega_{s, t}(x)
$$

exists and $\Omega_{s, t} \in \mathscr{C}_{R}^{\prime}$. Therefore, $\Omega_{s, t}$ has the form

$$
\begin{aligned}
\Omega_{s, t}(x)= & \ell \Omega_{s, t}(G)+\sum_{j=0}^{\infty}\left(\ell_{j}-\ell\right) \Omega_{s, t}\left(F^{(j)}\right) \\
& +\sum_{k=0}^{\infty}\left(h_{k}-\ell\right) \Omega_{s, t}\left(G^{(k)}\right) \\
& +\sum_{j=0}^{\infty} \sum_{k=0}^{\infty}\left(x_{j, k}-\ell_{j}-h_{k}+\ell\right) \Omega_{s, t}\left(F^{(j, k)}\right) .
\end{aligned}
$$

But $\Omega_{s, t}\left(F^{(j, k)}\right)=u_{j k}, \Omega_{s, t}\left(F^{(j)}\right)=u_{j 0}, \Omega_{s, t}\left(G^{(k)}\right)=u_{0 k}$, and $\Omega_{s, t}(G)=u$ by the conditions $\left(\mathrm{CR}_{2}\right)-\left(\mathrm{CR}_{5}\right)$, respectively. Hence

$$
\lim _{m, n \rightarrow \infty} \Omega_{m, n, s, t}(x)=\Omega(x)
$$

exists for each $x \in \mathscr{C}_{R}$ and $s, t=0,1,2, \ldots$ with

$$
\begin{aligned}
\Omega(x)= & \ell u+\sum_{j=0}^{\infty}\left(\ell_{j}-\ell\right) u_{j 0}+\sum_{k=0}^{\infty}\left(h_{k}-\ell\right) u_{0 k} \\
& +\sum_{j=0}^{\infty} \sum_{k=0}^{\infty}\left(x_{j, k}-\ell_{j}-h_{k}-\ell\right) u_{j k} .
\end{aligned}
$$

Since $\Omega_{m, n, s, t}(x) \in \mathscr{C}_{R}^{\prime}$ for each $m, n, s$, and $t$, it has the form

$$
\begin{aligned}
\Omega_{m, n, s, t}(x)= & \ell \Omega_{m, n, s, t}(G)+\sum_{j=0}^{\infty}\left(\ell_{j}-\ell\right) \Omega_{m, n, s, t}\left(F^{(j)}\right) \\
& +\sum_{k=0}^{\infty}\left(h_{k}-\ell\right) \Omega_{m, n, s, t}\left(G^{(k)}\right) \\
& +\sum_{j=0}^{\infty} \sum_{k=0}^{\infty}\left(x_{j, k}-\ell_{j}-h_{k}-\ell\right) \Omega_{m, n, s, t}\left(F^{(j, k)}\right) .
\end{aligned}
$$

It is easy to see from (21) and (22) that the convergence of $\left(\Omega_{m, n, s, t}(x)\right)$ to $\Omega(x)$ is uniform in $s, t$, since $\Omega_{m, n, s, t}(G) \rightarrow$ $u, \Omega_{m, n, s, t}\left(F^{(j)}\right) \rightarrow u_{j 0}, \Omega_{m, n, s, t}\left(G^{(k)}\right) \rightarrow u_{0 k}$, and $\Omega_{m, n, s, t}\left(F^{(j, k)}\right) \rightarrow u_{j k}(m, n \rightarrow \infty)$ uniformly in $s, t$. Therefore, $B$ is regularly $(\Lambda)$ almost conservative.

Let us recall the following lemma, which is proved by Mursaleen and Mohiuddine [25]. 
Lemma 7. Let $A(s, t)=\left(a_{m, n, j, k}(s, t)\right), s, t=0,1,2, \ldots$, be $a$ sequence of infinite matrices such that

(i) $\|A(s, t)\|<H<+\infty$ for all $s$, $t$; and

(ii) for each j, $k \lim _{m, n} a_{m, n, j, k}(s, t)=0$ uniformly in $s, t$.

Then

$$
\begin{aligned}
& \lim _{m, n} \sum_{j} \sum_{k} a_{m, n, j, k}(s, t) x_{j, k} \\
& \quad=0 \quad \text { uniformly in } s, t \text { for each } x \in \mathscr{L}^{\infty}
\end{aligned}
$$

if and only if

$$
\lim _{m, n} \sum_{j} \sum_{k}\left|a_{m, n, j, k}(s, t)\right|=0 \quad \text { uniformly in } s, t
$$

Theorem 8. A matrix $B=\left(b_{p, q, j, k}\right)$ is $(\Lambda)$ almost coercive if and only if

$\left(\mathrm{AC}_{1}\right)\|B\|=\sup _{p, q} \sum_{j, k}\left|b_{p, q, j, k}\right|<\infty$,

$\left(\mathrm{AC}_{2}\right) \lim _{m, n \rightarrow \infty} \alpha(m, n, s, t, j, k)=u_{j k}$, for each $j, k$ (uniformly in $s, t)$,

$\left(\mathrm{AC}_{3}\right) \lim _{m, n \rightarrow \infty} \sum_{j=1}^{\infty} \sum_{k=1}^{\infty}\left(1 / \lambda_{m} \mu_{n}\right) \mid \sum_{p \in J_{m}} \sum_{q \in I_{n}} b_{p+s, q+t, j, k}$ $-u_{j k} \mid=0$, uniformly in $s, t$.

In this case, the $\mathscr{F}_{\Lambda}$-limit of $B x$ is $\sum_{j=1}^{\infty} \sum_{k=1}^{\infty} u_{j k} x_{j, k}$ for every $\left(x_{j, k}\right) \in \mathscr{L}_{\infty}$.

Proof. Sufficiency. Assume that conditions $\left(\mathrm{AC}_{1}\right)-\left(\mathrm{AC}_{3}\right)$ hold. For any positive integers $J, K$

$$
\begin{aligned}
\sum_{j=1}^{J} \sum_{k=1}^{K}\left|u_{j k}\right| & =\sum_{j=1}^{J} \sum_{k=1}^{K} \lim _{m, n \rightarrow \infty} \frac{1}{\lambda_{m} \mu_{n}}\left|\sum_{p \in J_{m}} \sum_{q \in I_{n}} b_{p+s, q+t, j, k}\right| \\
& =\lim _{m, n \rightarrow \infty} \frac{1}{\lambda_{m} \mu_{n}} \sum_{j=1}^{J} \sum_{k=1}^{K}\left|\sum_{p \in J_{m}} \sum_{q \in I_{n}} b_{p+s, q+t, j, k}\right| \\
& \leq \limsup _{m, n} \frac{1}{\lambda_{m} \mu_{n}} \sum_{p \in J_{m}} \sum_{q \in I_{n}} \sum_{j=1}^{\infty} \sum_{k=1}^{\infty}\left|b_{p+s, q+t, j, k}\right| \\
& \leq\|B\| .
\end{aligned}
$$

This shows that $\sum_{j=1}^{\infty} \sum_{k=1}^{\infty}\left|u_{j k}\right|$ converges and that $\sum_{j=1}^{\infty} \sum_{k=1}^{\infty} u_{j k} x_{j, k}$ is defined for every double sequence $x=\left(x_{j, k}\right) \in \mathscr{L}_{\infty}$.
Let $\left(x_{j, k}\right)$ be any arbitrary bounded double sequence. For every positive integers $m, n$

$$
\begin{aligned}
& \left\|\sum_{j=1}^{\infty} \sum_{k=1}^{\infty}\left(\frac{1}{\lambda_{m} \mu_{n}} \sum_{p \in J_{m}} \sum_{q \in I_{n}} b_{p+s, q+t, j, k}-u_{j k}\right) x_{j, k}\right\| \\
& \quad=\left\|\sum_{j=1}^{\infty} \sum_{k=1}^{\infty}\left[\frac{1}{\lambda_{m} \mu_{n}} \sum_{p \in J_{m}} \sum_{q \in I_{n}}\left[b_{p+s, q+t, j, k}-u_{j k}\right]\right] x_{j, k}\right\| \\
& \leq \sup _{s, t}\left[\left|\sum_{j=1}^{\infty} \sum_{k=1}^{\infty}\left[\frac{1}{\lambda_{m} \mu_{n}} \sum_{p \in J_{m}} \sum_{q \in I_{n}}\left[b_{p+s, q+t, j, k}-u_{j k}\right]\right] x_{j, k}\right|\right] \\
& \leq\|x\| \sup _{s, t}\left[\frac{1}{\lambda_{m} \mu_{n}} \sum_{j=1}^{\infty} \sum_{k=1}^{\infty}\left|\sum_{p \in J_{m}} \sum_{q \in I_{n}}\left[b_{p+s, q+t, j, k}-u_{j k}\right]\right|\right] .
\end{aligned}
$$

Letting $p, q \rightarrow \infty$ and using condition $\left(\mathrm{AC}_{3}\right)$, we get

$$
\frac{1}{\lambda_{m} \mu_{n}} \sum_{j=1}^{\infty} \sum_{k=1}^{\infty} \sum_{p \in J_{m}} \sum_{q \in I_{n}} b_{p+s, q+t, j, k} x_{j, k} \longrightarrow \sum_{j=1}^{\infty} \sum_{k=1}^{\infty} u_{j k} x_{j, k} .
$$

Hence, $B x \in \mathscr{F}_{\Lambda}$ with $\mathscr{F}_{\Lambda^{-}} \lim B x=\sum_{j=1}^{\infty} \sum_{k=1}^{\infty} u_{j k} x_{j, k}$.

Necessity. Let $B$ be $(\Lambda)$ almost coercive matrix. This implies that a four-dimensional matrix $B$ is $(\Lambda)$ almost conservative; then we have conditions $\left(\mathrm{AC}_{1}\right)$ and $\left(\mathrm{AC}_{2}\right)$ from Theorem 6 . Now we have to show that $\left(\mathrm{AC}_{3}\right)$ holds.

Suppose that, for some $s, t$, we have

$$
\limsup _{m, n} \frac{1}{\lambda_{m} \mu_{n}} \sum_{j=1}^{\infty} \sum_{k=1}^{\infty}\left|\sum_{p \in J_{m}} \sum_{q \in I_{n}}\left[b_{p+s, q+t, j, k}-u_{j k}\right]\right|=N>0 .
$$

Since $\|B\|$ is finite, therefore $N$ is also finite. We observe that since $\sum_{j=1}^{\infty} \sum_{k=1}^{\infty}\left|u_{j k}\right|<+\infty$ and $B$ is $(\Lambda)$ almost coercive, the matrix $A=\left(a_{p, q, j, k}\right)$, where $a_{p, q, j, k}=b_{p, q, j, k}-u_{j k}$ is also $(\Lambda)$ almost coercive matrix. By an argument similar to that of Theorem 2.1 in [26] for single sequences, one can find $x \in \mathscr{L}_{\infty}$ for which $A x \notin \mathscr{F}_{\Lambda}$. This contradiction implies the necessity of $\left(\mathrm{AC}_{3}\right)$.

Now, we use Lemma 7 to show that this convergence is uniform in $s, t$. Let

$$
h_{m, n, j, k}(s, t)=\frac{1}{\lambda_{m} \mu_{n}} \sum_{p \in J_{m}} \sum_{q \in I_{n}}\left[b_{p, q, j, k}-u_{j k}\right]
$$

and let $H(s, t)$ be the matrix $\left(h_{m, n, j, k}(s, t)\right)$. It is easy to see that $\|H(s, t)\| \leq 2\|B\|$ for every $s, t$; and from condition $\left(\mathrm{AC}_{2}\right)$

$$
\lim _{m, n} h_{m, n, j, k}(s, t)=0 \quad \text { for each } j, k \text {, uniformly in } s, t \text {. }
$$

For any $x \in \mathscr{L}_{\infty}$,

$$
\lim _{m, n} \sum_{j=1}^{\infty} \sum_{k=1}^{\infty} h_{m, n, j, k}(s, t) x_{j, k}=\mathscr{F}_{\Lambda}-\lim B x-\sum_{j=1}^{\infty} \sum_{k=1}^{\infty} u_{j k} x_{j, k}
$$


and the limit exists uniformly in $s, t$, since $B x \in \mathscr{F}_{\Lambda}$. Moreover, this limit is zero since

$$
\begin{aligned}
& \left|\sum_{j=1}^{\infty} \sum_{k=1}^{\infty} h_{m, n, j, k}(s, t) x_{j, k}\right| \\
& \quad \leq \frac{\|x\| \sum_{j=1}^{\infty} \sum_{k=1}^{\infty}\left|\sum_{p \in J_{m}} \sum_{q \in I_{n}}\left[b_{p, q, j, k}-u_{j k}\right]\right|}{\lambda_{m} \mu_{n}} .
\end{aligned}
$$

Hence

$$
\lim _{m, n} \sum_{j=1}^{\infty} \sum_{k=1}^{\infty}\left|h_{m, m, j, k}(s, t)\right|=0 \quad \text { uniformly in } s, t .
$$

This shows that matrix $B=\left(b_{p, q, j, k}\right)$ satisfies condition $\left(\mathrm{AC}_{3}\right)$.

In the following theorem, we characterize the fourdimensional matrices of type $\left(\mathscr{L}_{1}, \mathscr{F}_{\Lambda}\right)$, where

$$
\mathscr{L}_{1}=\left\{x=\left(x_{j, k}\right): \sum_{j=0}^{\infty} \sum_{k=0}^{\infty}\left|x_{j, k}\right|<\infty\right\}
$$

the space of all absolutely convergent double series.

Theorem 9. A matrix $B \in\left(\mathscr{L}_{1}, \mathscr{F}_{\Lambda}\right)$ if and only if it satisfies the following conditions:

$$
\text { (i) } \sup _{m, n, s, t, j, k}\left|\left(1 / \lambda_{m} \mu_{n}\right) \sum_{p \in J_{m}} \sum_{q \in I_{n}} b_{p+s, q+t, j, k}\right|<\infty \text {, }
$$

and the condition $\left(\mathrm{CR}_{2}\right)$ of Theorem 6 holds.

Proof. Sufficiency. Suppose that conditions (i) and $\left(\mathrm{CR}_{2}\right)$ hold. For any double sequence $x=\left(x_{j, k}\right) \in \mathscr{L}_{1}$, we see that

$$
\lim _{m, n, \rightarrow \infty} \frac{1}{\lambda_{m} \mu_{n}} \sum_{j=1}^{\infty} \sum_{k=1}^{\infty} \sum_{p \in J_{m}} \sum_{q \in I_{n}} b_{p+s, q+t, j, k} x_{j, k}=\sum_{j=1}^{\infty} \sum_{k=1}^{\infty} u_{j k} x_{j, k}
$$

uniformly in $s, t$ and it also converges absolutely. Furthermore,

$$
\frac{1}{\lambda_{m} \mu_{n}} \sum_{j=1}^{\infty} \sum_{k=1}^{\infty} \sum_{p \in J_{m}} \sum_{q \in I_{n}} b_{p+s, q+t, j, k} x_{j, k}
$$

converges absolutely for each $m, n, s$, and $t$. Given $\epsilon>0$, there exist $j_{\circ}=j_{\circ}(\epsilon)$ and $k_{\circ}=k_{\circ}(\epsilon)$ such that

$$
\sum_{j>j_{\circ}} \sum_{k>k_{\circ}}\left|x_{j, k}\right|<\epsilon
$$

By the condition $\left(\mathrm{CR}_{2}\right)$, we can find $m_{\circ}, n_{\circ} \in \mathbb{N}$ such that

$$
\left|\sum_{j \leq j_{\circ}} \sum_{k \leq k_{\circ}}\left[\frac{1}{\lambda_{m} \mu_{n}} \sum_{p \in J_{m}} \sum_{q \in I_{n}} b_{p+s, q+t, j, k}-u_{j k}\right] x_{j, k}\right|<\infty,
$$

for all $m>m_{\mathrm{o}}$ and $n>n_{\mathrm{o}}$, uniformly in $s, t$. Now, by using the conditions (37), (38), and $\left(\mathrm{CR}_{2}\right)$, we get

$$
\begin{aligned}
\left|\sum_{j=1}^{\infty} \sum_{k=1}^{\infty}\left[\frac{1}{\lambda_{m} \mu_{n}} \sum_{p \in J_{m}} \sum_{q \in I_{n}} b_{p+s, q+t, j, k}-u_{j k}\right] x_{j, k}\right| \\
\leq\left|\sum_{j \leq j_{\circ}} \sum_{k \leq k_{\circ}}\left[\frac{1}{\lambda_{m} \mu_{n}} \sum_{p \in J_{m}} \sum_{q \in I_{n}} b_{p+s, q+t, j, k}-u_{j k}\right] x_{j, k}\right| \\
+\sum_{j>j_{\circ}} \sum_{k>k_{\circ}}\left|\frac{1}{\lambda_{m} \mu_{n}} \sum_{p \in J_{m}} \sum_{q \in I_{n}} b_{p+s, q+t, j, k}-u_{j k}\right|\left|x_{j, k}\right|,
\end{aligned}
$$

for all $m>m_{\circ}, n>n_{\circ}$ and uniformly in $s, t$. Hence (37) holds.

Necessity. Suppose that $B \in\left(\mathscr{L}_{1}, \mathscr{F}_{\Lambda}\right)$. The condition $\left(\mathrm{CR}_{2}\right)$ follows from the fact that $E \in \mathscr{L}_{1}$, where $E=\left(e^{(j, k)}\right)$ with $e^{(j, k)}=1$ for all $j, k$. To verify the condition (i), we define a continuous linear functional $L_{m, n, s, t}(x)$ on $\mathscr{L}_{1}$ by

$$
L_{m, n, s, t}(x)=\frac{1}{\lambda_{m} \mu_{n}} \sum_{j=1}^{\infty} \sum_{k=1}^{\infty} \sum_{p \in J_{m}} \sum_{q \in I_{n}} b_{p+s, q+t, j, k} x_{j, k} .
$$

Now

$$
\left|L_{m, n, s, t}(x)\right| \leq \sup _{j, k}\left|\frac{1}{\lambda_{m} \mu_{n}} \sum_{p \in J_{m}} \sum_{q \in I_{n}} b_{p+s, q+t, j, k}\right|\|x\|_{1}
$$

and hence

$$
\left\|L_{m, n, s, t}\right\| \leq \sup _{j, k}\left|\frac{1}{\lambda_{m} \mu_{n}} \sum_{p \in J_{m}} \sum_{q \in I_{n}} b_{p+s, q+t, j, k}\right| .
$$

For any fixed $j, k \in \mathbb{N}$, we define a double sequence $x=\left(x_{i, \ell}\right)$ by

$$
\begin{aligned}
& x_{i, \ell} \\
& = \begin{cases}\operatorname{sgn}\left(\frac{1}{\lambda_{m} \mu_{n}} \sum_{p \in J_{m}} \sum_{q \in I_{n}} b_{p+s, q+t, j, k}\right), & \text { for }(i, \ell)=(j, k), \\
0, & \text { for }(i, \ell) \neq(j, k) .\end{cases}
\end{aligned}
$$

Then $\|x\|_{1}=1$, and

$$
\begin{aligned}
\left|L_{m, n, s, t}(x)\right| & =\left|\frac{1}{\lambda_{m} \mu_{n}} \sum_{p \in J_{m}} \sum_{q \in I_{n}} b_{p+s, q+t, j, k} x_{j, k}\right| \\
& =\left|\frac{1}{\lambda_{m} \mu_{n}} \sum_{p \in J_{m}} \sum_{q \in I_{n}} b_{p+s, q+t, j, k}\right|\|x\|_{1},
\end{aligned}
$$

so that

$$
\left\|L_{m, n, s, t}\right\| \geq \sup _{j, k}\left|\frac{1}{\lambda_{m} \mu_{n}} \sum_{p \in J_{m}} \sum_{q \in I_{n}} b_{p+s, q+t, j, k}\right| .
$$


It follows from (42) and (45) that

$$
\left\|L_{m, n, s, t}\right\|=\sup _{j, k}\left|\frac{1}{\lambda_{m} \mu_{n}} \sum_{p \in J_{m}} \sum_{q \in I_{n}} b_{p+s, q+t, j, k}\right| .
$$

Since $B \in\left(\mathscr{L}_{1}, \mathscr{F}_{\Lambda}\right)$, we have

$$
\begin{aligned}
& \sup _{m, n, s, t}\left|L_{m, n, s, t}(x)\right| \\
& \quad=\sup _{m, n, s, t}\left|\frac{1}{\lambda_{m} \mu_{n}} \sum_{j=1}^{\infty} \sum_{k=1}^{\infty} \sum_{p \in J_{m}} \sum_{q \in I_{n}} b_{p+s, q+t, j, k} x_{j, k}\right|<\infty .
\end{aligned}
$$

Hence, by the uniform boundedness principle, we obtain

$$
\sup _{m, n, s, t}\left\|L_{m, n, s, t}(x)\right\|=\sup _{m, n, s, t, j, k}\left|\frac{1}{\lambda_{m} \mu_{n}} \sum_{p \in J_{m}} \sum_{q \in I_{n}} b_{p+s, q+t, j, k}\right|<\infty .
$$

\section{Conflict of Interests}

The authors declare that there is no conflict of interests regarding the publication of this paper.

\section{Acknowledgment}

This work was funded by the Deanship of Scientific Research (DSR), King Abdulaziz University, Jeddah, under Grant no. (130-100-D1434). The authors, therefore, acknowledge with thanks DSR technical and financial support.

\section{References}

[1] S. Banach, Théorie des Operations Lineaires, 1932.

[2] G. G. Lorentz, "A contribution to the theory of divergent sequences," Acta Mathematica, vol. 80, pp. 167-190, 1948.

[3] S. A. Mohiuddine, "An application of almost convergence in approximation theorems," Applied Mathematics Letters, vol. 24, no. 11, pp. 1856-1860, 2011.

[4] F. Móricz and B. E. Rhoades, "Almost convergence of double sequences and strong regularity of summability matrices," Mathematical Proceedings of the Cambridge Philosophical Society, vol. 104, no. 2, pp. 283-294, 1988.

[5] M. Mursaleen and S. A. Mohiuddine, "Banach limit and some new spaces of double sequences," Turkish Journal of Mathematics, vol. 36, no. 1, pp. 121-130, 2012.

[6] M. Başarir, "On the strong almost convergence of double sequences," Periodica Mathematica Hungarica, vol. 30, no. 3, pp. 177-181, 1995.

[7] F. Başar and M. Kirişçi, "Almost convergence and generalized difference matrix," Computers \& Mathematics with Applications, vol. 61, no. 3, pp. 602-611, 2011.

[8] F. Čnjalo, "Almost convergence of double subsequences," Filomat, vol. 22, no. 2, pp. 87-93, 2008.

[9] K. Kayaduman and C. Çakan, "The cesáro core of double sequences," Abstract and Applied Analysis, vol. 2011, Article ID 950364, 9 pages, 2011.
[10] Mursaleen, "Almost strongly regular matrices and a core theorem for double sequences," Journal of Mathematical Analysis and Applications, vol. 293, no. 2, pp. 523-531, 2004.

[11] Mursaleen and O. H. H. Edely, "Almost convergence and a core theorem for double sequences," Journal of Mathematical Analysis and Applications, vol. 293, no. 2, pp. 532-540, 2004.

[12] M. Zeltser, M. Mursaleen, and S. A. Mohiuddine, "On almost conservative matrix methods for double sequence spaces," Publicationes Mathematicae Debrecen, vol. 75, no. 3-4, pp. 387399, 2009.

[13] A. Pringsheim, "Zur theorie der zweifach unendlichen zahlenfolgen," Mathematische Annalen, vol. 53, no. 3, pp. 289-321, 1900.

[14] B. Altay and F. Başar, "Some new spaces of double sequences," Journal of Mathematical Analysis and Applications, vol. 309, no. 1, pp. 70-90, 2005.

[15] H. J. Hamilton, “Transformations of multiple sequences," Duke Mathematical Journal, vol. 2, no. 1, pp. 29-60, 1936.

[16] S. A. Mohiuddine and A. Alotaibi, "Some spaces of double sequences obtained through invariant mean and related concepts," Abstract and Applied Analysis, vol. 2013, Article ID 507950, 11 pages, 2013.

[17] Mursaleen and S. A. Mohiuddine, "Double $\sigma$-multiplicative matrices," Journal of Mathematical Analysis and Applications, vol. 327, no. 2, pp. 991-996, 2007.

[18] M. Mursaleen and S. A. Mohiuddine, "On $\sigma$-conservative and boundedly $\sigma$-conservative four-dimensional matrices," Computers \& Mathematics with Applications, vol. 59, no. 2, pp. 880885, 2010.

[19] P. Schaefer, "Infinite matrices and invariant means," Proceedings of the American Mathematical Society, vol. 36, pp. 104-110, 1972.

[20] G. M. Robison, "Divergent double sequences and series," Transactions of the American Mathematical Society, vol. 28, no. 1, pp. 50-73, 1926.

[21] J. P. King, "Almost summable sequences," Proceedings of the American Mathematical Society, vol. 17, pp. 1219-1225, 1966.

[22] P. Schaefer, "Matrix transformations of almost convergent sequences," Mathematische Zeitschrift, vol. 112, pp. 321-325, 1969.

[23] F. Başar and I. Solak, "Almost-coercive matrix transformations," Rendiconti di Matematica e delle sue Applicazioni, vol. 11, no. 2, pp. 249-256, 1991.

[24] F. Móricz, "Extensions of the spaces $c$ and $c_{0}$ from single to double sequences," Acta Mathematica Hungarica, vol. 57, no. 1-2, pp. 129-136, 1991.

[25] M. Mursaleen and S. A. Mohiuddine, "Regularly $\sigma$-conservative and $\sigma$-coercive four dimensional matrices," Computers \& Mathematics with Applications, vol. 56, no. 6, pp. 1580-1586, 2008.

[26] C. Eizen and G. Laush, "Infinite matrices and almost convergence," Mathematica Japonica, vol. 14, pp. 137-143, 1969. 


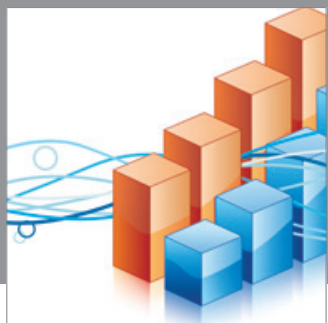

Advances in

Operations Research

mansans

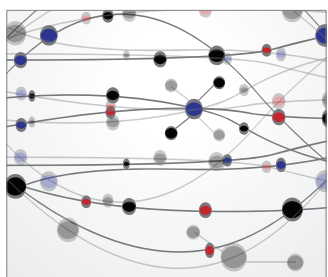

The Scientific World Journal
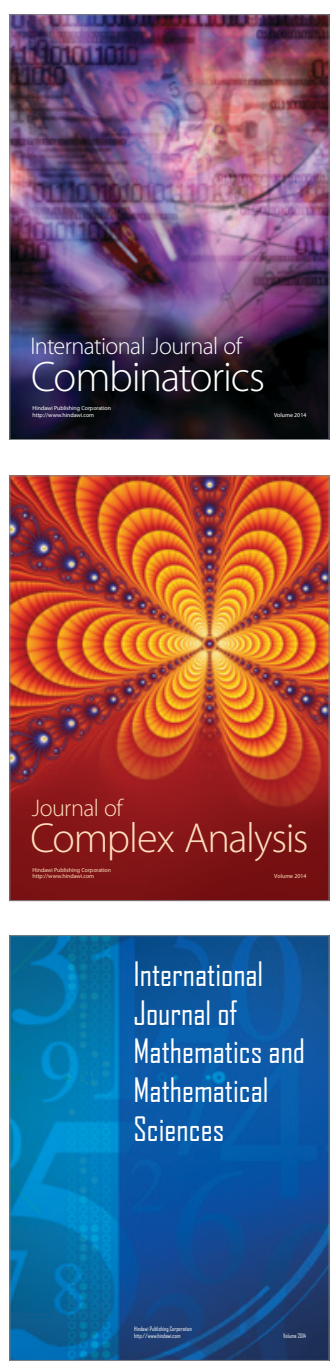
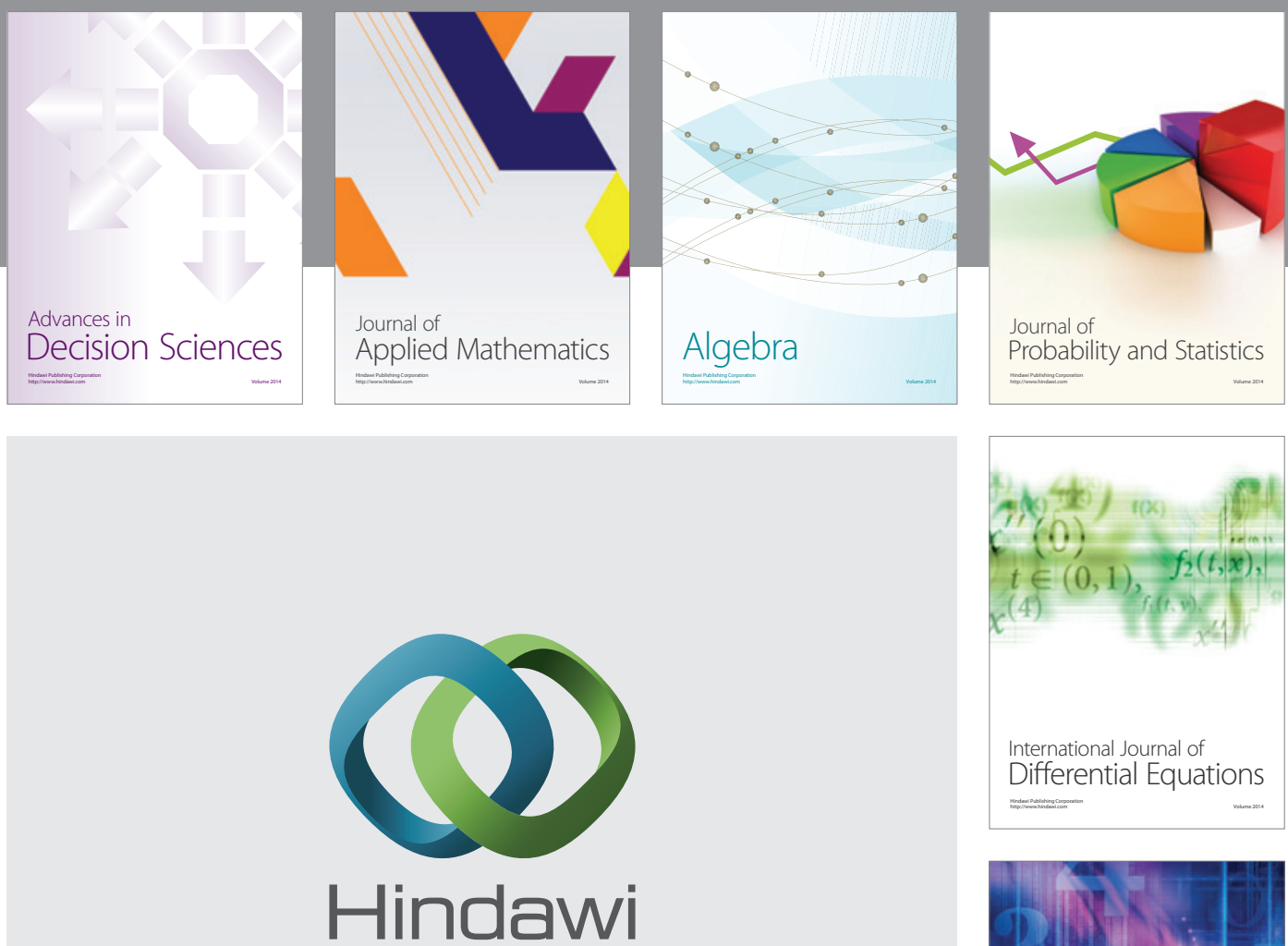

Submit your manuscripts at http://www.hindawi.com
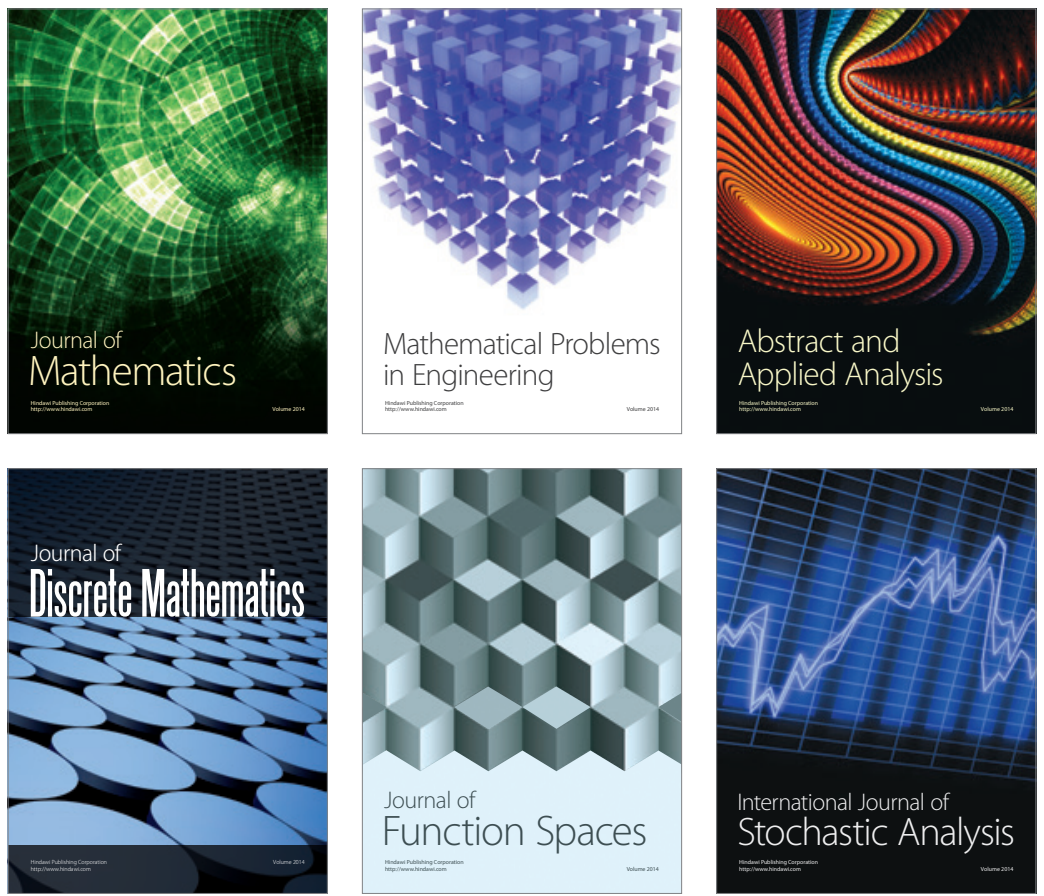

Journal of

Function Spaces

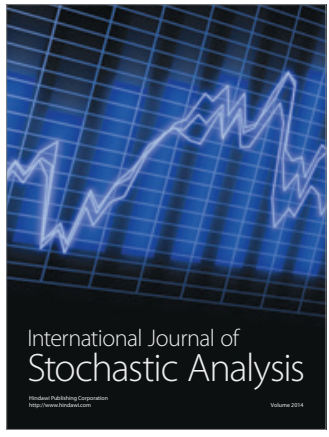

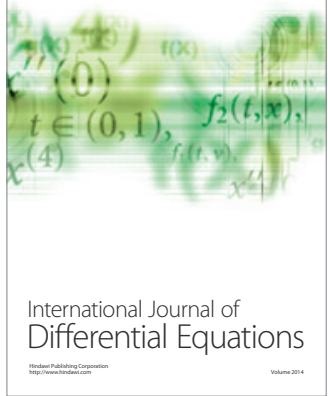
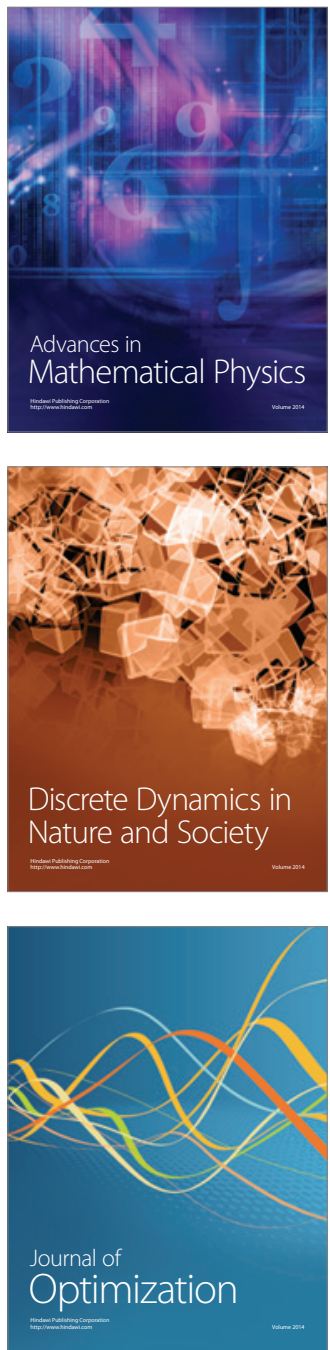